\title{
French "Carto PMAi" project: \\ exposure risk assessment of workers and public population to the EMP
}

ERELL LEOCAT AND CHRISTINE DENEUVILLERS

\section{OPPBTP}

Presenting Author: erell.leocat@oppbtp.fr

The national project entitled Carto PMAi involves leading an exploratory campaign for assessing the potential exposure of worker and public populations to Elongate Mineral Particles of interest (EMPi). These particles are the asbestiform and nonasbestiform varieties of the six regulated asbestos minerals and to four other mineral fibers known as carcinogen for human. The aim is to give the relevant data to the Ministries of Health, of Labor and of Environment allowing them to set up legal provisions proportionally to the risk if the population is exposed to EMPi. For this exploratory campaign, the concerned professional sectors are the most emissive and the most frequent situations.

The French government mandated the OPPBTP (Professional Body of Prevention in Public Work and Building Domain) to conduct this measurement campaign. The "Carto" process that is also used for other campaigns implies single operating procedures, measurement monitoring and scientific validation. Several national scientific organizations and asbestos testing laboratories take part to the project. Its first step is to build reliable protocols by testing them in the field to measure EMPi in materials and in the air. The second phase is the measurement campaign in construction areas, including quarries and earthwork in natural environment. The measurement campaign is still in progress. 